\title{
Circulating adipsin is associated with asymptomatic carotid atherosclerosis in obese adults
}

Jinhua Zhang ${ }^{1 \dagger}$, Fei Teng ${ }^{2,4 \dagger}$, Lingling Pan ${ }^{3 \dagger}$, Dan Guo ${ }^{2}$, Jianfang Liu², Kangli Li ${ }^{2}$, Youwen Yuan², Wenyuan $\mathrm{Li}^{2^{*}}$ and Huijie Zhang ${ }^{2,4,5^{*}}$

\begin{abstract}
Background: Adipsin has been identified as a secreted adipokine that plays a critical pathogenic role in metabolic disorders. However, it is not clear regarding the association of circulating adipsin with cardiovascular disease (CVD). This study will explore the association between circulating adipsin and asymptomatic carotid atherosclerosis in Chinese obese adults.

Methods: A total of 483 obese adult subjects (aged 40 years or older) were enrolled in this study. Serum adipsin concentrations and carotid intima-media thickness (CIMT) were measured to determine these associations.

Results: Individuals with increased CIMT and asymptomatic carotid atherosclerosis had lower levels of circulating adipsin than controls (both $p<0.05$ ). The prevalence of asymptomatic carotid atherosclerosis was significantly higher in subjects with lower levels of serum adipsin than those with higher values ( $42.5 \%$ vs. $36.7 \%, p<0.05)$. Notably, subjects in the lowest quartile of serum adipsin were 1.94 times $(p=0.059)$ more likely to have increased CIMT and 2.91 times ( $p=0.03$ ) more likely to have asymptomatic carotid atherosclerosis than those in the highest quartile in multivariable logistic regression analyses, adjusting for age, gender, current smoking, alcohol consumption, physical activity, BMI, systolic BP, fasting glucose, total cholesterol, HDL-C, and HOMA-IR. However, such associations with circulating adipsin were not noted for atherosclerotic plaque.
\end{abstract}

Conclusions: These findings suggest that circulating adipsin concentrations are a potential marker of risks of increased CIMT and asymptomatic carotid atherosclerosis in obese Chinese adults.

Keywords: Adipsin, Obesity, Carotid intima-media thickness, Cardiovascular disease, Asymptomatic carotid atherosclerosis

*Correspondence: liwy666@163.com; huijiezhang2005@126.com ${ }^{\dagger}$ Jinhua Zhang, Fei Teng and Lingling Pan have equally contributed to this work

${ }^{2}$ Department of Endocrinology and Metabolism, Nanfang Hospital, Southern Medical University, 1838 North Guangzhou Road, Guangzhou 510515, China

Full list of author information is available at the end of the article

\section{Introduction}

Cardiovascular disease (CVD) is the leading cause of morbidity and mortality worldwide [1], which gained worldwide attention. It includes myocardial infarction, hypertension, atherosclerosis, vascular disease, and stroke [2], affecting approximately 290 million people in China [3, 4]. However, almost half of patients who died from CVD can have no clinical symptoms of CVD [5]. Subclinical CVD is a broad term that includes carotid atherosclerosis and arterial stiffness strongly associated 
with future CVD events [6, 7]. Asymptomatic carotid atherosclerosis is the most common type of subclinical CVD [8]. Identification of risk factors for the development of symptomatic carotid atherosclerosis could prevent and delay the progress of CVD. Numerous risk factors have been well established, including age, smoking, physical inactivity, obesity, hypertension, diabetes, and hyperlipidemia $[9,10]$. Excess adiposity is strongly associated with the risk of CVD in the general population [11].

It has been proposed that obesity-associated insulin resistance and low-grade inflammation may largely account for the development of CVD [12, 13]. However, the mechanisms by which insulin resistance and obesity promote CVD risk remain uncertain. Adipose tissue has been identified as an energy storage organ. Meanwhile, it also plays as an essential endocrine organ secreting adipokines [14, 15]. Several adipokines, including Leptin, fetuin-A, and adiponectin, are associated with risk of obesity, type 2 diabetes (T2DM), metabolic syndrome (MetS), and CVD [13, 16-20].

Adipsin, a member of the serine protease gene family, is recently identified as a novel secreted adipokine, playing an important role in modulating systemic energy metabolism [21]. Previous studies have shown that adipsin plays a significant role in controlling blood glucose levels and insulin sensitivity [22-24]. Banoy et al. reported that adipsin plays a pivotal role in preserving beta cells and protecting from type 2 diabetes in diabetic mice and humans through splitting complement factor $\mathrm{B}$ in the alternative complement pathway and catalyzing the formation of complement component C3a [23]. Levels of adipsin are decreased in patients with T2DM [25]. As described above, the associations between adipsin and metabolic disorders have been previously discussed. Given the connection between obesity-associated metabolic disorder and the risk of CVD, circulating adipsin might be a candidate marker of CVD risk. However, there is little evidence regarding the association between circulating adipsin and CVD in the community cohort study. In this study, we aimed to explore the association between circulating adipsin and the risk of asymptomatic carotid atherosclerosis in Chinese obese adults.

\section{Methods}

\section{Study participants}

Between April 2011 and December 2013, 1536 obese subjects aged 40 years or older were recruited from the community in China after physical examination [26]. Obesity was defined as an elevated waist circumference (> $90 \mathrm{~cm}$ in males or $80 \mathrm{~cm}$ in females). Subjects with the following conditions were excluded from this study: acute or chronic viral hepatitis, drug-induced liver diseases, obstructive biliary diseases, Wilson's disease, auto-immune disease, total parenteral nutrition, cancer, or known hypothyroidism or hyperthyroidism. A total of 520 participants were randomly chosen to receive carotid ultrasonography for the measurement of carotid intimamedia thickness. Participants with missing data for serum adipsin levels were further excluded in this study. In total, our final analysis included 483 participants.

All subjects provided written informed consent. The study protocol was approved by the Institutional Review Board of the First Affiliated Hospital of Xiamen University and Nanfang Hospital of Southern Medical University. The methods were carried out in accordance with the approved guidelines.

\section{Clinical and biochemical measurements}

A spring scale and a vertical ruler measure were used to obtain bodyweight and height measurements, respectively. Body mass index (BMI) measures total body adiposity. Waist circumference was measured at the level of the umbilicus. Blood pressure (BP) was assessed in triplicate using an electronic sphygmomanometer (OMRON Company). Body fat mass was determined using the HOLOGIC whole body DXA system (Hologic Inc., Bedford, MA). Each measurement was performed at least three times, and the mean value was used for analysis.

Each subject underwent 75 -g oral glucose tolerance tests and blood biochemical measurements a $12 \mathrm{~h}$ fast. Plasma glucose, high-density lipoprotein cholesterol (HDL-c), triglycerides (TG), and total cholesterol (TC) were measured by enzymatic colorimetric methods with a Hitachi 7450 analyzer (Hitachi, Tokyo, Japan). Lowdensity lipoprotein cholesterol (LDL-C) was calculated by Friedewald's formula. Fasting plasma glucose concentrations and 2-h glucose concentrations were measured using the glucose oxidase method. Serum insulin concentrations were measured using electrochemiluminescence immunoassay (Roche Elecsys Insulin Test, Roche Diagnostics, Mannheim, Germany). Insulin resistance was estimated using HOMA of insulin resistance (HOMA-IR).

\section{Measurement of carotid intima-media thickness}

The Carotid intima-media thickness (CIMT) was performed and analyzed by a single trained sonographer using a high-resolution B-mode tomographic ultrasound system (Philips-ATL HDI-5000, Philips Medical Systems, Bothell, WA, USA) [27]. The far wall of both left and right common carotid arteries at $1.0 \mathrm{~cm}$ proximal to the bifurcation were scanned. The mean of the maximum CIMT reading of right and left far walls for common, bulb, and internal segments were calculated [27]. Plaque was defined as an echogenic focal structure encroaching the vessel lumen with at least $50 \%$ greater thickness than 
that found in surrounding areas. All these measurements were repeated three times.

\section{Serum Adipsin measurement}

Serum adipsin concentrations were measured by using enzyme-linked immunosorbent assay (ELISA) kits (AssayPro, St. Charles, MO, USA) according to the manufacturer's instructions. The assay has been shown to be highly sensitive to human adipsin with a sensitivity of $0.0021 \mathrm{ug} / \mathrm{ml}$. The linear range of the standard was $0.001875-0.120 \mathrm{ug} / \mathrm{ml}$, and the intra and inter assay variations were both less than $10 \%$.

\section{Statistical analysis}

Data are presented as means \pm standard deviation (S.D) for normally distributed variables, median (interquartile range) for variables that were not normally distributed, and numbers (percentages) for the categorical variables. Data that were not normally distributed were logarithmically transformed before analysis. The subjects were classified into four quartiles according to their serum level of adipsin.

Chi-square test or logistic regression models were used to examine differences in categorical variables in different study groups. Analyses of covariance were performed using general linear models (GLM) to test differences in study variables between different quartiles of serum adipsin. The Benjamini-Hochberg method was used to correct the p-values for the multiple comparisons. Multivariable logistic regression models were used to examine the association of serum adipsin levels with risks of increased CIMT, adjusted for age, gender, smoking, alcohol consumption, physical activity, BMI, systolic BP, glucose, triglyceride, HDL-c, HOMA-IR, and body fat mass. Increased CIMT was defined as the average CIMT $\geq 0.8 \mathrm{~mm}$ [28]. Asymptomatic carotid atherosclerosis was defined as increased CIMT or the presence of atherosclerotic plaque. Two-sided values of $p<0.05$ were considered statistically significant. All statistical analyses were performed with SAS version 9.3 (SAS Institute, Cary, NC).

\section{Results}

The demographic, clinical, and laboratory characteristics were shown in Table 1 . Obese subjects with increased CIMT had higher levels of current smokers, systolic blood pressure, total cholesterol, LDL-c, fasting glucose, and visceral fat compared with those without increased CIMT (all $p<0.05$ ). Of interest, subjects with increased CIMT had lower serum adipsin levels than subjects without increased CIMT $(25.7 \pm 7.1 \mathrm{ng} /$ $\mathrm{ml}$ vs. $27.4 \pm 10.7 \mathrm{ng} / \mathrm{ml}, p=0.002)$. Besides, patients with atherosclerotic plaques had higher total cholesterol and LDL-c than subjects without atherosclerotic plaque $(p<0.05)$. There are no differences in serum adipsin in subjects without atherosclerotic plaque compared to subjects with atherosclerotic plaque. Additionally, subjects with asymptomatic carotid atherosclerosis had an unfavorable metabolic profile, including higher levels of total cholesterol, LDL-c, and visceral fat. Importantly, subjects with asymptomatic carotid atherosclerosis had lower serum adipsin levels than subjects without asymptomatic carotid atherosclerosis $(26.4 \pm 9.4 \mathrm{ug} / \mathrm{ml}$ vs. $27.2 \pm 10.2$ $\mathrm{ug} / \mathrm{ml}, p=0.004)$.

The clinical and biochemical characteristics by quartiles of serum adipsin levels were shown in Table 2 . systolic BP, diastolic BP, total cholesterol, triglyceride, LDL-c, HDL-c, fasting glucose, and visceral fat showed no significant differences among the four quartiles of serum adipsin levels, adjusted with age and gender. Compared to subjects in the lowest quartile of serum adipsin levels, those in the highest quartile had significantly higher levels of BMI, waist circumference, body fat mass, and lower levels of postprandial glucose and HOMAIR (both $\mathrm{p}<0.05$ ). Additionally, CIMT was reduced gradually with the increase of serum adipsin $(p=0.009)$. Interestingly, the prevalence of asymptomatic carotid atherosclerosis was significantly higher in subjects with lower levels of serum adipsin than those with higher values $(42.5 \%$ vs. $36.7 \%, p<0.05)$. However, the prevalence of atherosclerotic plaque showed no significant difference across quartiles of serum adipsin levels.

As shown in Fig. 1, CIMT was reduced gradually with the increase of serum adipsin in obese subjects $(p=0.036)$. Serum adipsin was significantly lower in patients with subclinical cardiovascular disease than nonsubclinical cardiovascular disease subjects $(p=0.004)$. However, compared with subjects without atherosclerotic plaques, there was no significant difference between the two groups.

Multivariable logistic regression was utilized to estimate adjusted odds ratios (ORs) for the association of serum adipsin levels with increased CIMT, atherosclerotic plaque, and asymptomatic carotid atherosclerosis are shown in Table 3. The risk of increased CIMT was positively associated with HOMA-IR $(p=0.043)$ and visceral fat $(p<0.001)$. Meanwhile, serum adipsin showed a significant association with increased CIMT after adjustment for age and gender $(p=0.003)$. Importantly, subjects in the second quartile and the third quartile of serum adipsin were 3.536 times $(p=0.001)$ and 2.443 times $(p=0.006)$ more likely to have increased CIMT than those in the highest quartile. Furthermore, the risk of increased CIMT was still significantly higher in subjects in the lower quartile of serum adipsin than those in the highest quartile, even after adjusting for 
Table 1 Clinical and biochemical characteristics of obese subjects

\begin{tabular}{|c|c|c|c|c|c|c|c|c|c|}
\hline \multirow[t]{2}{*}{ Variables } & \multicolumn{3}{|c|}{ Increased CIMT } & \multicolumn{3}{|c|}{ Atherosclerotic plaque } & \multicolumn{3}{|c|}{ Asymptomatic carotid atherosclerosis* } \\
\hline & Yes & No & P-value ${ }^{\S}$ & Yes & No & P-value ${ }^{\S}$ & Yes & No & P-value ${ }^{\S}$ \\
\hline Sample size & 138 & 345 & & 116 & 367 & & 209 & 274 & \\
\hline Age (years) & $56.0 \pm 6.2$ & $53.4 \pm 7.4$ & $<0.001$ & $58.1 \pm 6.1$ & $52.9 \pm 7.0$ & $<0.001$ & $56.7 \pm 6.3$ & $52.2 \pm 7.2$ & $<0.001$ \\
\hline $\begin{array}{l}\text { Gender (male } \\
\mathrm{n}, \%)\end{array}$ & $56(40.6)$ & $74(21.5)$ & $<0.001$ & $41(35.3)$ & $89(24.3)$ & 0.019 & $75(35.9)$ & $55(20.1)$ & $<0.001$ \\
\hline $\mathrm{BMI}\left(\mathrm{kg} / \mathrm{m}^{2}\right)$ & $27.6 \pm 2.9$ & $27.1 \pm 2.9$ & 0.081 & $26.9 \pm 3.0$ & $27.3 \pm 2.9$ & 0.225 & $27.3 \pm 3.0$ & $27.2 \pm 2.9$ & 0.599 \\
\hline $\begin{array}{l}\text { Waist circum- } \\
\text { ference }(\mathrm{cm})\end{array}$ & $95.1 \pm 7.1$ & $93.9 \pm 6.8$ & 0.755 & $94.0 \pm 6.5$ & $94.3 \pm 7.0$ & 0.079 & $94.6 \pm 6.9$ & $93.9 \pm 6.9$ & 0.550 \\
\hline $\begin{array}{l}\text { Current smok- } \\
\text { ers }(n, \%)\end{array}$ & $31(22.5)$ & $43(12.5)$ & 0.006 & $20(17.2)$ & $54(14.7)$ & 0.511 & $41(19.6)$ & $33(12.0)$ & 0.022 \\
\hline $\begin{array}{l}\text { Systolic BP } \\
\text { (mmHg) }\end{array}$ & $134.8 \pm 17.5$ & $127.4 \pm 15.2$ & 0.001 & $133.7 \pm 18.5$ & $128.2 \pm 15.2$ & 0.148 & $132.7 \pm 17.6$ & $127.1 \pm 14.7$ & 0.084 \\
\hline $\begin{array}{l}\text { Diastolic BP } \\
(\mathrm{mmHg})\end{array}$ & $80.0 \pm 10.5$ & $77.1 \pm 9.8$ & 0.052 & $78.7 \pm 10.6$ & $77.7 \pm 9.9$ & 0.733 & $78.7 \pm 10.4$ & $77.3 \pm 9.8$ & 0.594 \\
\hline $\begin{array}{l}\text { Triglycerides } \\
\text { (mmol/L) }\end{array}$ & $\begin{array}{l}1.83(1.31- \\
2.35)\end{array}$ & $\begin{array}{l}1.51(1.13- \\
2.12)\end{array}$ & 0.682 & $\begin{array}{l}1.65(1.19- \\
2.14)\end{array}$ & $\begin{array}{l}1.58(1.17- \\
2.20)\end{array}$ & 0.780 & $\begin{array}{l}1.70(1.20- \\
2.23)\end{array}$ & $\begin{array}{l}1.54(1.12- \\
2.14)\end{array}$ & 0.591 \\
\hline $\begin{array}{l}\text { Total choles- } \\
\text { terol (mmol/L) }\end{array}$ & $6.07 \pm 1.07$ & $5.64 \pm 0.94$ & $<0.001$ & $6.03 \pm 1.10$ & $5.67 \pm 0.95$ & 0.014 & $5.96 \pm 1.04$ & $5.61 \pm 0.94$ & 0.003 \\
\hline $\begin{array}{l}\text { LDL-choles- } \\
\text { terol (mmol/L) }\end{array}$ & $4.00 \pm 1.00$ & $3.68 \pm 0.96$ & 0.009 & $4.06 \pm 1.10$ & $3.68 \pm 0.92$ & 0.014 & $3.96 \pm 0.99$ & $3.63 \pm 0.94$ & 0.012 \\
\hline $\begin{array}{l}\text { HDL-choles- } \\
\text { terol (mmol/L) }\end{array}$ & $1.28 \pm 0.22$ & $1.32 \pm 0.27$ & 0.542 & $1.30 \pm 0.24$ & $1.31 \pm 0.26$ & 0.687 & $1.28 \pm 0.23$ & $1.33 \pm 0.27$ & 0.204 \\
\hline $\begin{array}{l}\text { Fasting glu- } \\
\text { cose (mmol/L) }\end{array}$ & $5.75 \pm 0.91$ & $5.53 \pm 0.70$ & 0.032 & $5.65 \pm 1.01$ & $5.57 \pm 0.68$ & 0.911 & $5.65 \pm 0.82$ & $5.55 \pm 0.74$ & 0.789 \\
\hline $\begin{array}{l}\text { 2-h glucose } \\
\text { (mmol/L) }\end{array}$ & $8.07 \pm 2.34$ & $7.95 \pm 2.26$ & 0.734 & $8.05 \pm 2.59$ & $7.97 \pm 2.18$ & 0.702 & $7.97 \pm 2.29$ & $8.00 \pm 2.28$ & 0.406 \\
\hline HOMA-IR & $\begin{array}{l}2.91(2.26- \\
3.87)\end{array}$ & $\begin{array}{l}2.83(1.84- \\
4.08)\end{array}$ & 0.147 & $\begin{array}{l}2.62(1.90- \\
4.07)\end{array}$ & $\begin{array}{l}2.97(1.95- \\
4.01)\end{array}$ & 0.736 & $\begin{array}{l}2.72(1.97- \\
3.86)\end{array}$ & $\begin{array}{l}2.98(1.93- \\
4.11)\end{array}$ & 0.965 \\
\hline $\begin{array}{l}\text { Serum adipsin } \\
\text { (ug/ml) }\end{array}$ & $5.13 \pm 1.43$ & $5.47 \pm 2.14$ & 0.002 & $5.50 \pm 2.20$ & $5.33 \pm 1.89$ & 0.475 & $5.28 \pm 1.88$ & $5.44 \pm 2.04$ & 0.004 \\
\hline $\begin{array}{l}\text { Body fat } \\
\operatorname{mass}(\mathrm{kg})\end{array}$ & $22.9 \pm 5.2$ & $23.2 \pm 4.5$ & 0.432 & $22.3 \pm 4.7$ & $23.4 \pm 4.7$ & 0.228 & $5.29 \pm 1.88$ & $5.44 \pm 2.04$ & 0.876 \\
\hline $\begin{array}{l}\text { Visceral fat } \\
\left(\mathrm{cm}^{2}\right)\end{array}$ & $144.3 \pm 45.7$ & $115.2 \pm 40.1$ & $<0.001$ & $127.6 \pm 42.5$ & $122.3 \pm 44.1$ & 0.159 & $134.2 \pm 44.8$ & $115.4 \pm 41.2$ & 0.036 \\
\hline
\end{tabular}

${ }^{*}$ Asymptomatic carotid atherosclerosis is defined as increased CIMT or presence of atherosclerotic plaque

$\mathrm{CIMT}=$ Carotid intima-media thickness; BMI = body mass index; HOMA-IR=homeostasis model assessment of insulin resistance;

Data are presented as the mean \pm SD or median (interquartile range) or numbers (percentages)

$\S$ Adjusted for age and gender

age, gender, current smoking, alcohol consumption, and physical activity. In addition, this relationship remained significant after further adjusting for age, gender, current smoking, alcohol consumption, physical activity, systolic BP, glucose, total cholesterol, triglyceride, and HDL-c. The risks of asymptomatic carotid atherosclerosis were reduced by $29.6 \%$ per 1 SD increase in serum adipsin levels (log-transformed). Notably, the ORs for asymptomatic carotid atherosclerosis remained significant OR [ $(95 \%$ CI) 0.705 (0.567-0.876), $\mathrm{p}=0.002]$, even after adjusting for age, sex, current smoking, alcohol consumption, and physical activity. After further adjustment for systolic BP, glucose, total cholesterol, triglyceride, and HDL-c, the relationship between serum adipsin and asymptomatic carotid atherosclerosis remained significant [OR (95\% CI) 0.665 (0.532-0.832), $\mathrm{p}<0.001]$. Similarly, subjects in the lower quartiles also showed a significantly elevated risk for asymptomatic carotid atherosclerosis compared to those in the fourth quartile (all $p<0.05$ ). However, such associations with circulating adipsin were not noted for atherosclerotic plaque.

\section{Discussion}

Adipsin is recently identified as a novel adipokine that may modulate systemic energy metabolism and play a critical pathogenic role in obesity-associated metabolic 
Table 2 Clinical and biochemical characteristics by quartiles of serum adipsin levels

\begin{tabular}{|c|c|c|c|c|c|}
\hline \multirow[t]{2}{*}{ Variables } & \multicolumn{4}{|c|}{ Serum adipsin level } & \multirow[t]{2}{*}{ P-value for trend ${ }^{\S}$ : } \\
\hline & Q1 & Q2 & Q3 & Q4 & \\
\hline Sample size & 120 & 121 & 122 & 120 & \\
\hline Serum adipsin (ug/ml) & $3.52 \pm 0.61$ & $4.53 \pm 0.35$ & $5.49 \pm 0.44$ & $7.96 \pm 2.01$ & $<0.001$ \\
\hline Age (years) & $53.0 \pm 7.6$ & $53.5 \pm 7.0$ & $54.0 \pm 7.2$ & $56.0 \pm 6.5 \neq$ & 0.007 \\
\hline Gender (male n, \%) & $32(26.7)$ & $33(27.3)$ & $33(27.1)$ & $32(26.7)$ & 0.990 \\
\hline $\mathrm{BMI}\left(\mathrm{kg} / \mathrm{m}^{2}\right)$ & $26.7 \pm 2.8$ & $27.1 \pm 2.8$ & $27.6 \pm 3.0$ & $27.6 \pm 3.1 \dagger$ & 0.036 \\
\hline Waist circumference (cm) & $92.8 \pm 6.6$ & $93.6 \pm 6.9$ & $94.8 \pm 6.9+$ & $95.7 \pm 6.9 \neq$ & 0.007 \\
\hline Current smokers (n, \%) & $17(14.2)$ & 16(13.2) & $23(18.9)$ & $18(15.0)$ & 0.633 \\
\hline Systolic BP (mmHg) & $128.1 \pm 15.3$ & $129.6 \pm 17.3$ & $128.8 \pm 13.7$ & $131.6 \pm 18.3$ & 0.705 \\
\hline Diastolic BP (mmHg) & $77.2 \pm 9.6$ & $78.0 \pm 10.9$ & $78.0 \pm 9.6$ & $78.4 \pm 10.3$ & 0.821 \\
\hline Triglycerides (mmol/L) & $1.50(1.13-2.14)$ & $1.57(1.24-2.23)$ & $1.60(1.14-2.16)$ & $1.70(1.22-2.25)$ & 0.268 \\
\hline Total cholesterol (mmol/L) & $5.67 \pm 1.06$ & $5.71 \pm 0.94$ & $5.81 \pm 0.99$ & $5.84 \pm 0.99$ & 0.737 \\
\hline LDL- cholesterol (mmol/L) & $3.66 \pm 1.08$ & $3.67 \pm 0.92$ & $3.89 \pm 0.99$ & $3.86 \pm 0.90$ & 0.248 \\
\hline HDL-cholesterol (mmol/L) & $1.32 \pm 0.26$ & $1.29 \pm 0.28$ & $1.32 \pm 0.23$ & $1.31 \pm 0.26$ & 0.644 \\
\hline Fasting glucose (mmol/L) & $5.64 \pm 0.89$ & $5.67 \pm 1.03$ & $5.50 \pm 0.50$ & $5.55 \pm 0.53$ & 0.183 \\
\hline 2-h glucose (mmol/L) & $8.37 \pm 2.55$ & $8.12 \pm 2.49$ & $7.63 \pm 1.80 \dagger$ & $7.83 \pm 2.17 \dagger$ & 0.027 \\
\hline HOMA-IR & $2.97(1.82-4.06)$ & $3.10(2.14-4.52)$ & $2.58(1.78-3.64)$ & $2.80(2.08-4.00)$ & 0.015 \\
\hline Body fat mass(kg) & $22.2 \pm 4.7$ & $22.9 \pm 4.3$ & $23.6 \pm 4.8+$ & $23.9 \pm 4.9 \neq$ & 0.017 \\
\hline Visceral fat $\left(\mathrm{cm}^{2}\right)$ & $116.3 \pm 38.8$ & $126.6 \pm 50.7$ & $124.0 \pm 44.9$ & $127.4 \pm 39.0$ & 0.302 \\
\hline CIMT (mm): & $0.74 \pm 0.16$ & $0.76 \pm 0.17$ & $0.73 \pm 0.15$ & $0.71 \pm 0.13 \dagger$ & 0.009 \\
\hline Atherosclerotic plaque $(n, \%)$ & $31(25.8)$ & $28(23.1)$ & $25(20.5)$ & $32(26.7)$ & 0.259 \\
\hline Asymptomatic carotid atherosclerosis* & $51(42.5)$ & $60(50.0)$ & $54(44.3)$ & $44(36.7)$ & 0.017 \\
\hline
\end{tabular}

\section{* Asymptomatic carotid atherosclerosis is defined as increased CIMT or presence of atherosclerotic plaque}

$\mathrm{CIMT}=$ Carotid intima-media thickness; $\mathrm{BMI}=$ body mass index; HOMA-IR=homeostasis model assessment of insulin resistance;

Data are presented as the mean \pm SD or median (interquartile range) or numbers (percentages)

$\S$ Adjusted for age and gender

${ }^{\dagger}$ Adjusted $\mathrm{P}<0.05$ compared with $\mathrm{Q} 1$ of serum adipsin

${ }^{\ddagger}$ Adjusted $\mathrm{P}<0.01$ compared with $\mathrm{Q} 1$ of serum adipsin

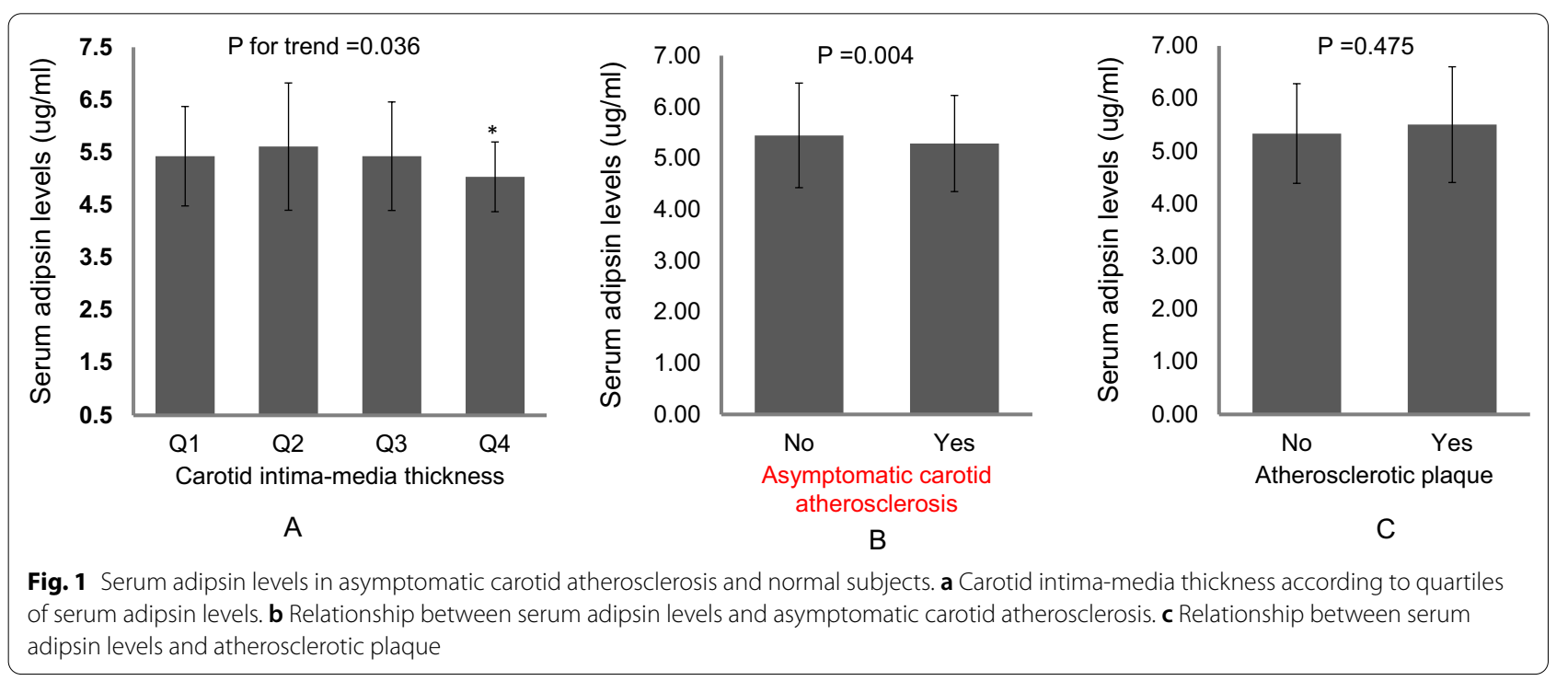


Table 3 Adjusted odds ratios (ORs) with associated 95\% confidence interval (Cl) for asymptomatic carotid atherosclerosis

\begin{tabular}{|c|c|c|c|c|c|c|c|c|c|}
\hline \multirow[t]{2}{*}{ Variables } & \multicolumn{3}{|c|}{ Increased CIMT } & \multicolumn{3}{|c|}{ Atherosclerotic plaque } & \multicolumn{3}{|c|}{$\begin{array}{l}\text { Asymptomatic carotid } \\
\text { atherosclerosis* }\end{array}$} \\
\hline & OR & $95 \% \mathrm{Cl}$ & P-value & OR & $95 \% \mathrm{Cl}$ & P-value & OR & $95 \% \mathrm{Cl}$ & P-value \\
\hline \multicolumn{10}{|l|}{ Model 1} \\
\hline BMI $\left(\mathrm{kg} / \mathrm{m}^{2}\right)$ & 1.244 & $0.992-1.560$ & 0.059 & 0.789 & $0.606-1.026$ & 0.077 & 1.048 & $0.846-1.299$ & 0.669 \\
\hline Waist circumference (cm) & 1.037 & $0.827-1.300$ & 0.754 & 0.752 & $0.577-0.979$ & 0.034 & 0.932 & $0.752-1.156$ & 0.523 \\
\hline HOMA-IR & 1.491 & $1.013-2.192$ & 0.043 & 1.040 & $0.685-1.579$ & 0.854 & 1.098 & $0.764-1.577$ & 0.613 \\
\hline Body fat mass (Kg) & 1.086 & $0.864-1.365$ & 0.482 & 0.797 & $0.613-1.035$ & 0.089 & 0.991 & $0.798-1.231$ & 0.937 \\
\hline Visceral fat $\left(\mathrm{cm}^{2}\right)$ & 1.736 & $1.374-2.194$ & $<0.001$ & 0.853 & $0.666-1.092$ & 0.206 & 1.271 & $1.022-1.581$ & 0.031 \\
\hline Serum adipsin (ug/ml) & 0.691 & $0.541-0.882$ & 0.003 & 0.877 & $0.696-1.107$ & 0.269 & 0.704 & $0.567-0.875$ & 0.002 \\
\hline \multicolumn{10}{|l|}{ Serum adipsin (ug/ml) } \\
\hline (Quartile 1 vs. Quartile 4) & 1.762 & $0.916-3.390$ & 0.090 & 1.435 & $0.764-2.696$ & 0.261 & 2.002 & $1.120-3.579$ & 0.019 \\
\hline (Quartile 2 vs. Quartile 4) & 3.536 & $1.898-6.588$ & $<0.001$ & 1.144 & $0.608-2.152$ & 0.677 & 2.528 & $1.431-4.469$ & 0.001 \\
\hline (Quartile 3 vs. Quartile 4) & 2.443 & $1.299-4.595$ & 0.006 & 0.839 & $0.437-1.609$ & 0.597 & 1.813 & $1.022-3.216$ & 0.042 \\
\hline \multicolumn{10}{|l|}{ Model 2} \\
\hline BMI $\left(\mathrm{kg} / \mathrm{m}^{2}\right)$ & 1.251 & $0.996-1.570$ & 0.054 & 0.784 & $0.602-1.021$ & 0.071 & 1.049 & $0.846-1.301$ & 0.660 \\
\hline Waist circumference (cm) & 1.043 & $0.831-1.310$ & 0.714 & 0.759 & $0.582-0.990$ & 0.042 & 0.936 & $0.754-1.162$ & 0.551 \\
\hline HOMA-IR & 1.501 & $1.015-2.218$ & 0.042 & 1.011 & $0.806-1.269$ & 0.922 & 1.095 & $0.761-1.577$ & 0.625 \\
\hline Body fat mass $(\mathrm{Kg})$ & 1.095 & $0.871-1.378$ & 0.437 & 0.801 & $0.616-1.041$ & 0.097 & 0.998 & $0.803-1.240$ & 0.983 \\
\hline Visceral fat $\left(\mathrm{cm}^{2}\right)$ & 1.777 & $1.400-2.255$ & $<0.001$ & 0.850 & $0.662-1.092$ & 0.205 & 1.284 & $1.030-1.600$ & 0.026 \\
\hline Serum adipsin (ug/ml) & 0.690 & $0.540-0.883$ & 0.003 & 0.879 & $0.697-1.109$ & 0.277 & 0.705 & $0.567-0.876$ & 0.002 \\
\hline \multicolumn{10}{|l|}{ Serum adipsin (ug/ml) } \\
\hline (Quartile 1 vs. Quartile 4) & 1.788 & $0.930-3.441$ & 0.082 & 1.426 & $0.758-2.682$ & 0.271 & 2.023 & $1.131-3.619$ & 0.018 \\
\hline (Quartile 2 vs. Quartile 4) & 3.562 & $1.907-6.652$ & $<0.001$ & 1.124 & $0.596-2.119$ & 0.718 & 2.559 & $1.444-4.534$ & 0.001 \\
\hline (Quartile 3 vs. Quartile 4) & 2.417 & $1.278-4.569$ & 0.007 & 0.806 & $0.417-1.557$ & 0.521 & 1.798 & $1.008-3.204$ & 0.047 \\
\hline \multicolumn{10}{|l|}{ Model 3} \\
\hline BMI $\left(\mathrm{kg} / \mathrm{m}^{2}\right)$ & 1.131 & $0.884-1.446$ & 0.328 & 0.711 & $0.535-0.944$ & 0.019 & 0.972 & $0.772-1.223$ & 0.807 \\
\hline Waist circumference (cm) & 0.979 & $0.770-1.244$ & 0.860 & 0.724 & $0.550-0.954$ & 0.022 & 0.893 & $0.713-1.118$ & 0.323 \\
\hline HOMA-IR & 1.220 & $0.742-2.007$ & 0.433 & 0.995 & $0.586-1.691$ & 0.985 & 1.068 & $0.671-1.700$ & 0.780 \\
\hline Body fat mass (Kg) & 1.040 & $0.817-1.323$ & 0.751 & 0.775 & $0.591-1.016$ & 0.065 & 0.970 & $0.773-1.217$ & 0.791 \\
\hline Visceral fat $\left(\mathrm{cm}^{2}\right)$ & 1.674 & $1.302-2.153$ & $<0.001$ & 0.767 & $0.584-1.007$ & 0.057 & 1.210 & $0.955-1.532$ & 0.115 \\
\hline Serum adipsin (ug/ml) & 0.655 & $0.509-0.843$ & 0.001 & 0.848 & $0.670-1.072$ & 0.168 & 0.665 & $0.532-0.832$ & $<0.001$ \\
\hline \multicolumn{10}{|l|}{ Serum adipsin (ug/ml) } \\
\hline (Quartile 1 vs. Quartile 4) & 1.935 & $0.976-3.836$ & 0.059 & 1.528 & $0.799-2.923$ & 0.200 & 2.267 & $1.236-4.158$ & 0.008 \\
\hline (Quartile 2 vs. Quartile 4) & 4.248 & $2.195-8.224$ & $<0.001$ & 1.221 & $0.637-2.339$ & 0.548 & 2.963 & $1.630-5.386$ & $<0.001$ \\
\hline (Quartile 3 vs. Quartile 4) & 2.742 & $1.411-5.328$ & 0.003 & 0.809 & $0.413-1.584$ & 0.537 & 1.910 & $1.049-3.475$ & 0.034 \\
\hline
\end{tabular}

${ }^{*}$ Asymptomatic carotid atherosclerosis is defined as increased CIMT or presence of atherosclerotic plaque

$\mathrm{OR}=$ odds ratio; $\mathrm{Cl}=$ confidence interval; $\mathrm{MI}=$ body mass index $\mathrm{CIMT}=$ Carotid intima-media thickness; $\mathrm{BMI}=$ body mass index; $\mathrm{HOMA}-\mathrm{IR}=$ homeostasis model assessment of insulin resistance;

Model 1: adjusted for age, gender

Model 2: adjusted for model $1+$ smoking, alcohol consumption, and physical activity

Model 3: adjusted for model 2+SBP, glucose, total cholesterol, triglyceride, and HDL-c

diseases [21, 23, 29]. In the present study, we provide novel evidence that circulating adipsin concentrations were significantly reduced in subjects with asymptomatic carotid atherosclerosis and were inversely associated with increased CIMT in obese adults. However, serum adipsin was not significantly associated with atherosclerotic plaques. These findings indicate that circulating adipsin could be a potential marker in the development of CVD.

Adipsin is secreted mainly by adipose tissue and enhanced significantly during the progress of adipose tissue synthesis, which plays a vital role in obesity-related metabolic diseases, including type 2 diabetes and NAFLD $[21,30,31]$. Recently, adipsin has been identified as 
playing a pivotal role in preserving beta cells through controlling the complement pathway and generation of complement component $\mathrm{C} 3 \mathrm{a}$ in diabetic mice and associates with protection from type 2 diabetes in humans [21, 23]. Some studies reported that $\beta$ cell failure and poor glycemic control in Type 2 diabetes were inversely correlated to circulating adipsin levels [23, 32]. In addition, Liu et al. reported that serum adipsin concentrations were negatively associated with circulating cardiac markers [33]. It also has been reported that circulating adipsin levels were higher in overweight/obesity subjects and associated with increased cardiovascular risk in patients with polycystic ovary syndrome [34, 35]. Our study found that serum adipsin levels positively correlated with waist circumference and body fat, whereas another study reported that circulating adipsin levels were associated with subcutaneous fat rather than visceral adiposity [23]. Furthermore, Jun-Sing Wang and colleague reported that serum adipsin levels were negatively associated with insulin resistance in 320 subjects with various degrees of glucose intolerance, especially in subjects with a BMI $\geq 25 \mathrm{~kg} / \mathrm{m}^{2}$ or prediabetes [24]. Consistently, our findings indicated that those in the highest quartile of serum adipsin levels had significantly lower levels of postprandial glucose and HOMA-IR. These findings suggest that adipsin may be a potential marker for metabolic syndrome.

Previous studies indicated that adipsin was associated with protection from type 2 diabetes and lower levels of metabolic risk in humans [21, 23]. In a cohort of 9771 healthy middle-aged men, higher plasma levels of adipsin was associated with an increased 10-year risk of ischemic stroke [36]. However, the role of serum adipsin levels in predicting incident CVD is still not conclusive. In particular, the relationship between circulating adipsin and subclinical atherosclerosis in human subjects remains largely unclear. Our present study provides novel evidence that circulating adipsin concentrations were significantly reduced in obese Chinese adults with asymptomatic carotid atherosclerosis and were inversely associated with increased CIMT. In addition, the relationship between circulating adipsin concentrations and CIMT may be modified by age. These findings suggest that serum levels of adipsin could be a potential marker in the development of CVD.

There are several potential reasons for circulating adipsin as a potential marker in the development of CVD in obese adults. First, serum adipsin level was negatively correlated with Type 2 diabetes [23] and other risk factors of CVD, including postprandial glucose and HOMAIR. Thereby, it is no surprise that circulating adipsin concentrations could predict the development of CVD. Second, it can regulate glucose metabolism and vascular function. In this regard, circulating adipsin may regulate the complement replacement pathway to catalyze the production of the $\mathrm{C} 3 \mathrm{a}$ and modulate vascular endothelial function $(21,37,38)$. Therefore, circulating adipsin may be a potential marker for the risks of CVD in obese subjects. Prospective cohort studies are needed to confirm this finding and elucidate the potential underlying mechanisms.

This community-based, cross-sectional study provided an opportunity to determine the role of circulating adipsin in predicting the development of CVD and increased CIMT. There are certain limitations of our study. First, the population consisted of only obese adults with a relatively limited sample size in the present study. It is reported that adipsin has a correlation with adiposity and metabolic risk factors, which may confound the association of adipsin with metabolic diseases and the ability of adipsin to predict clinical outcomes. However, we found independent associations between circulating adipsin and outcomes even after adjusting for multiply potential confounders. Then we need further studies to determine the role of circulating adipsin in the development of CVD in the general population. Second, due to this study's cross-sectional observational design, we could not determine whether there was a true causal relationship between circulating adipsin concentrations and the development of CVD. Therefore, further studies with long-term follow-up periods and larger sample sizes are warranted. Third, the study was limited to the Chinese population. Furthermore, the aim of our current study was defined as subclinical atherosclerosis rather than clinical cardiovascular events. Further studies need to determine the role of serum adipsin in predicting CVD events in different populations.

\section{Conclusions}

In conclusion, these findings indicate that circulating adipsin concentrations are inversely associated with the risk of increased CIMT and asymptomatic carotid atherosclerosis in obese Chinese adults, suggesting that circulating adipsin concentrations may be a potential marker in the development of CVD and would be useful to improve strategies for CVD prevention.

\section{Abbreviations \\ CVD: Cardiovascular disease; CIMT: Carotid intima-media thickness; T2DM: Type 2 diabetes mellitus; MetS: Metabolic syndrome; BMI: Body mass index; BP: Blood pressure; HDL-c: High-density lipoprotein cholesterol; TG: Triglycerides; TC: Total cholesterol; LDL-c: Low-density lipoprotein cholesterol; HOMA-IR: Homeostasis model assessment of insulin resistance; GLM: General linear models.}

\section{Acknowledgements}

None. 


\section{Authors' contributions}

$J Z, F T, W L$ and $H Z$ generated the hypothesis, directed implementation, and wrote the manuscript. LP, DG, JL and JZ contributed to analytic strategy and statistical analyses. $\mathrm{HZ}, \mathrm{KL}$ and $\mathrm{YY}$ supervised the field activities and data collection and edited the manuscript. $\mathrm{HZ}$ is the guarantor of this work and, as such, had full access to all the data in the study and takes responsibility for the integrity of the data and the accuracy of the data analysis. All authors take responsibility for all aspects of the reliability and freedom from bias of the data presented and their discussed interpretation. All authors read and approved the final manuscript.

\section{Funding}

This study was supported by the National Key Research and Development Project (No. 2018YFA0800404), Natural Science Foundation and KeyArea Research and Development Program of Guangdong Province (Nos. 2018B030311031 and 2019B020227004), Young Foundation of Fujian Provincial Education Department (No. JAT170711), Clinical Research Startup Program of Southern Medical University by High-level University Construction Funding of Guangdong Provincial Department of Education (No. LC2019ZD010), and the National Natural Science Foundation of China (Nos. 81970736 and 81700743). Dr. Huijie Zhang was partially supported by Distinguished Young Scholars Training Program of Nanfang Hospital.

\section{Availability of data and materials}

Data are available on request from the corresponding author.

\section{Declarations}

\section{Ethics approval and consent to participate}

All subjects provided written informed consent. The study protocol was approved by the Institutional Review Board of the First Affiliated Hospital of Xiamen University and Nanfang Hospital of Southern Medical University.

\section{Consent for publication}

The patients gave written informed consent for clinical data use and for publication.

\section{Competing interests}

The authors declares that there is no conflict of interest.

\section{Author details}

${ }^{1}$ Key Laboratory of Functional and Clinical Translational Medicine, Department of General Medicine, Xiamen Medical College, Xiamen, China. ${ }^{2}$ Department of Endocrinology and Metabolism, Nanfang Hospital, Southern Medical University, 1838 North Guangzhou Road, Guangzhou 510515, China. ${ }^{3}$ Department of Endocrinology and Metabolism, Tongji Hospital, Tongji University, Shanghai, China. ${ }^{4}$ The First Affiliated Hospital of Xiamen University, Xiamen, China. ${ }^{5}$ Department of Medical Imaging Center, Nanfang Hospital, Southern Medical University, Guangzhou, China.

Received: 19 March 2021 Accepted: 13 October 2021 Published online: 25 October 2021

\section{References}

1. Smith S. Screening for high-risk cardiovascular disease: a challenge for the guidelines: comment on "systematic review of guidelines on cardiovascular risk assessment: which recommendations should clinicians follow for a cardiovascular health check?" Arch Internal Med. 2010;170(1):40-2.

2. Ryan C, Kirby B. Psoriasis is a systemic disease with multiple cardiovascular and metabolic comorbidities. Dermatol Clin. 2015;33(1):41-55.

3. Chen W, Gao R, Liu L, Zhu M-L, Wang W, Wang Y-J, et al. China cardiovascular diseases report 2015: a summary. J Geriatr Cardiol. 2017;14(1):1-10.

4. Yang G, Wang Y, Zeng Y, Gao GF, Liang X, Zhou M, et al. Rapid health transition in China, 1990-2010: findings from the Global Burden of Disease Study 2010. Lancet. 2013;381(9882):1987-2015.

5. Ni H, Coady S, Rosamond W, Folsom A, Chambless L, Russell S, et al. Trends from 1987 to 2004 in sudden death due to coronary heart disease: the Atherosclerosis Risk in Communities (ARIC) study. Am Heart J. 2009;157(1):46-52.

6. Greenland P, Alpert J, Beller G, Benjamin E, Budoff M, Fayad Z, et al. 2010 ACCF/AHA guideline for assessment of cardiovascular risk in asymptomatic adults: a report of the American College of Cardiology Foundation/American Heart Association Task Force on Practice Guidelines. J Am Coll Cardiol. 2010;56(25):e50-103.

7. Dietz L, Matthews K. Depressive symptoms and subclinical markers of cardiovascular disease in adolescents. J Adolescent Health. 2011;48(6):579-84

8. Cao J, Arnold A, Manolio T, Polak J, Psaty B, Hirsch C, et al. Association of carotid artery intima-media thickness, plaques, and C-reactive protein with future cardiovascular disease and all-cause mortality: the Cardiovascular Health Study. Circulation. 2007;116(1):32-8.

9. Goff DC Jr, Lloyd-Jones DM, Bennett G, Coady S, D'Agostino RB Sr, Gibbons R, et al. 2013 ACC/AHA guideline on the assessment of cardiovascular risk: a report of the American College of Cardiology/American Heart Association Task Force on Practice Guidelines. J Am Coll Cardiol. 2014;63(25 Pt B):2935-59.

10. Lakka HM, Laaksonen DE, Lakka TA, Niskanen LK, Kumpusalo E, Tuomilehto J, et al. The metabolic syndrome and total and cardiovascular disease mortality in middle-aged men. JAMA. 2002;288(21):2709-16.

11. Lavie CJ, De Schutter A, Parto P, Jahangir E, Kokkinos P, Ortega FB, et al. Obesity and prevalence of cardiovascular diseases and prognosis-the obesity paradox updated. Prog Cardiovasc Dis. 2016;58(5):537-47.

12. Ormazabal V, Nair S, Elfeky O, Aguayo C, Salomon C, Zuniga FA. Association between insulin resistance and the development of cardiovascular disease. Cardiovasc Diabetol. 2018;17(1):122.

13. Chait $A$, den Hartigh LJ. Adipose tissue distribution, inflammation and its metabolic consequences, including diabetes and cardiovascular disease. Front Cardiovasc Med. 2020;7:22.

14. Galic S, Oakhill JS, Steinberg GR. Adipose tissue as an endocrine organ. Mol Cell Endocrinol. 2010;316(2):129-39.

15. Jung UJ, Choi MS. Obesity and its metabolic complications: the role of adipokines and the relationship between obesity, inflammation, insulin resistance, dyslipidemia and nonalcoholic fatty liver disease. Int J Mol Sci. 2014;15(4):6184-223.

16. Lee MW, Lee M, Oh KJ. Adipose tissue-derived signatures for obesity and type 2 diabetes: adipokines, batokines and microRNAs. J Clin Med. 2019;8(6).

17. Francisco V, Ruiz-Fernandez C, Pino J, Mera A, Gonzalez-Gay MA, Gomez R, et al. Adipokines: linking metabolic syndrome, the immune system, and arthritic diseases. Biochem Pharmacol. 2019;165:196-206.

18. Berg AH, Scherer PE. Adipose tissue, inflammation, and cardiovascular disease. Circ Res. 2005;96(9):939-49.

19. Meex RCR, Watt MJ. Hepatokines: linking nonalcoholic fatty liver disease and insulin resistance. Nat Rev Endocrinol. 2017;13(9):509-20.

20. Fasshauer M, Blüher M. Adipokines in health and disease. Trends Pharmacol Sci. 2015;36(7):461-70.

21. Lo JC, Ljubicic S, Leibiger B, Kern M, Leibiger IB, Moede T, et al. Adipsin is an adipokine that improves beta cell function in diabetes. Cell. 2014;158(1):41-53.

22. Miner J, Byatt J, Baile C, Krivi G. Adipsin expression and growth in rats as influenced by insulin and somatotropin. Physiol Behav. 1993;54(2):207-12.

23. Gomez-Banoy N, Guseh JS, Li G, Rubio-Navarro A, Chen T, Poirier $B$, et al. Adipsin preserves beta cells in diabetic mice and associates with protection from type 2 diabetes in humans. Nat Med. 2019;25(11):1739-47.

24. Wang JS, Lee WJ, Lee IT, Lin SY, Lee WL, Liang KW, et al. Association between serum adipsin levels and insulin resistance in subjects with various degrees of glucose intolerance. J Endocr Soc. 2019;3(2):403-10.

25. Li N, Zhao Y, Yue Y, Chen L, Yao Z, Niu W. Liraglutide ameliorates palmitate-induced endothelial dysfunction through activating AMPK and reversing leptin resistance. Biochem Biophys Res Commun. 2016;478(1):46-52.

26. Pan LL, Zhang HJ, Huang ZF, Sun Q, Chen Z, Li ZB, et al. Intrahepatic triglyceride content is independently associated with chronic kidney disease in obese adults: a cross-sectional study. Metabolism. 2015;64(9):1077-85. 
27. Jiang J, Lin M, Xu Y, Shao J, Li X, Zhang H, et al. Circulating neuregulin 4 levels are inversely associated with subclinical cardiovascular disease in obese adults. Sci Rep. 2016;6:36710.

28. Zhao Y, Evans MA, Allison MA, Bertoni AG, Budoff MJ, Criqui MH, et al. Multisite atherosclerosis in subjects with metabolic syndrome and diabetes and relation to cardiovascular events: the multi-ethnic study of atherosclerosis. Atherosclerosis. 2019;282:202-9.

29. Rosen B, Cook K, Yaglom J, Groves D, Volanakis J, Damm D, et al. Adipsin and complement factor D activity: an immune-related defect in obesity. Science. 1989;244(4911):1483-7.

30. Polyzos SA, Kountouras J, Mantzoros CS. Adipokines in nonalcoholic fatty liver disease. Metabolism. 2016:65(8):1062-79.

31. Miner J, Hahn K, Spurlock M, Staten NJ. Expression and complement d activity of porcine adipsin. Protein Expr Purif. 2001;23(1):14-21.

32. Legakis I, Mantzouridis T, Bouboulis G, Chrousos GP. Reciprocal changes of serum adispin and visfatin levels in patients with type 2 diabetes after an overnight fast. Arch Endocrinol Metab. 2016;60(1):76-8.

33. Liu W, Jiang L, Chen J, Gao C, Zhou J, Zhou J, et al. Association of adipokines with blood pressure, arterial elasticity and cardiac markers in dialysis patients: cross-sectional analysis of baseline data from a cohort study. Nutr Metab. 2017;14:34.

34. Gursoy Calan O, Calan M, Yesil Senses P, Unal Kocabas G, Ozden E, Sari KR, et al. Increased adipsin is associated with carotid intima media thickness and metabolic disturbances in polycystic ovary syndrome. Clin Endocrinol (Oxf). 2016;85(6):910-7.

35. Derosa G, Fogari E, D'Angelo A, Bianchi L, Bonaventura A, Romano D, et al Adipocytokine levels in obese and non-obese subjects: an observational study. Inflammation. 2013;36(4):914-20.

36. Prugger C, Luc G, Haas B, Arveiler D, Machez E, Ferrieres J, et al. Adipocytokines and the risk of ischemic stroke: the PRIME Study. Ann Neurol. 2012;71(4):478-86.

37. Sole S, Srinivasan B. Aqueous extract of tamarind seeds selectively increases glucose transporter-2, glucose transporter-4, and islets' intracellular calcium levels and stimulates $\beta$-cell proliferation resulting in improved glucose homeostasis in rats with streptozotocin-induced diabetes mellitus. Nutr Res. 2012;32(8):626-36.

38. Singh S, Vats P, Suri S, Shyam R, Kumria M, Ranganathan S, et al. Effect of an antidiabetic extract of Catharanthus roseus on enzymic activities in streptozotocin induced diabetic rats. J Ethnopharmacol. 2001;76(3):269-77.

\section{Publisher's Note}

Springer Nature remains neutral with regard to jurisdictional claims in published maps and institutional affiliations.
Ready to submit your research? Choose BMC and benefit from:

- fast, convenient online submission

- thorough peer review by experienced researchers in your field

- rapid publication on acceptance

- support for research data, including large and complex data types

- gold Open Access which fosters wider collaboration and increased citations

- maximum visibility for your research: over $100 \mathrm{M}$ website views per year

At BMC, research is always in progress.

Learn more biomedcentral.com/submissions 